\title{
Performance Analysis of Wide Area Network Communications using Discrete Event Simulation Tools
}

\author{
M. Golshani, G. A. Taylor, I. Pisica, P. M. Ashton, C. Chen, J. Liu, and J. Lin
}

\begin{abstract}
Operation of power systems has become more complicated and therefore encounters more challenges. In this regard, Wide Area Monitoring System (WAMS) as a state-of-the-art technology provides an effective method for large scale power grid monitoring, protection and control. Phasor Measurement Units (PMUs) measure power system parameters including frequency, voltage and current phasors with high accuracy and transmit the measured values online to a central location called Phasor Data Concentrator (PDC). Then the collected information can be exploited by smart grid applications. Critical to the operation of such a system is a high speed communication infrastructure. The performance of communications links has direct impact on the ability to meet specific smart grid applications requirements. In this paper we present performance evaluation of the WAMS communications infrastructure with regard to latency and End-to-End (EtE) delay from PMUs to PDC. An actual WAMS as installed on the transmission system of Great Britain (GB) is modelled using both proprietary and open source Discrete Event Simulators (DES), OPNET and OMNeT++. Comparisons will be also drawn between the two simulation environments approaches and the results obtained.
\end{abstract}

Index Terms-Discrete Event Simulation, OMNeT++, OPNET, PMU, WAMS

\section{INTRODUCTION}

$\mathrm{T}$ HE Penetration of renewable energy sources has been increased due to fast depletion of conventional resources and transition to a low carbon energy system. The UK government has set targets regarding proportion of energy to be generated from renewables by 2020 . Based on this targets, the electricity transmission system operator of Great Britain,

M. Golshani, G. A. Taylor, I. Pisica, and P. M. Ashton are with the Brunel Institute of Power Systems (BIPS), Brunel University, London, UK (email: mohammad.golshani@brunel.ac.uk, gareth.taylor@brunel.ac.uk, ioana.pisica@brunel.ac.uk, phillip.ashton@brunel.ac.uk).

C. Chen and J. Liu are with Sichuan University, Chengdu, China (email: chen.chen2@brunel.ac.uk, liujy@scu.edu.cn).

J. Lin is with Tsinghua University, Beijing, China (email: linjin@tsinghua.edu.cn).
National Grid (NG), has developed a "Gone-Green" scenario for the future energy mix. This scenario outlines the anticipated rise in generating capacity from wind, $3.8 \mathrm{GW}$ in $2010 / 11$ to $26.8 \mathrm{GW}$ in 2020/21 [1]. However, wind energy is highly intermittent, unstable, and unpredictable in nature, which makes the electric grid more dynamic, distributed, deregulated, and therefore complex [2]. In addition, there are restrictions in upgrading and expanding the transmission lines proportional to the growing generations, which leads the power grid operating closer to its parameters limits. In order to operate the power system reliably in this condition, smart and advanced monitoring system is a key technology [3].

Traditionally, Supervisory Control and Data Acquisition (SCADA) systems were designed for monitoring of the power systems by polling the Remote Terminal Units (RTUs) at all the substations. However, the gathering of data is done at a slow rate of every few seconds so the monitoring is relatively static and infrequent. In addition, SCADA data are not consistently time-synchronized and shared widely across the network. Therefore, SCADA does not provide operators with real-time and wide-area visibility. Recently, synchronized phasor measurement technique offers a complementary way for monitoring of the power systems. A PMU-based WAMS is a system in which PMUs, from geographically distributed areas, measure grid parameters with high sample rate, high accuracy and relative to a known time-reference. These measurements are then communicated to a central location where they are used for monitoring purposes or by assessment applications [4].

The first WAMS was deployed in the GB Electricity National Control Centre (ENCC) in 1998. This system was developed by Psymetrix and is running PhasorPoint application to carry out continuous analysis of the dynamics of the GB system [5]. In order to effectively monitor the inter-area modes between Scotland and England, information is required from the respective centres of inertia for both areas. In the absence of data from the Scottish system, as shown in Fig. 1, one PMU was installed in the North of England, close to the Anglo-Scottish boundary and another PMU was installed close to the centre of inertia for England and Wales. By comparing 


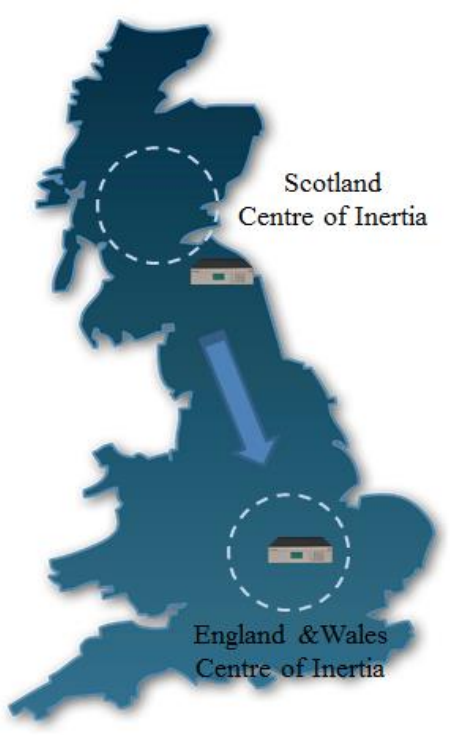

Fig. 1. PMUs placement for monitoring of the Anglo-Scottish interconnection

the level of oscillations between the two locations it is possible to determine whether the source of the oscillation is in the North or the South of the network. Also the provided information enables operators to identify whether the oscillation damping on the system has fallen below the predefined stability margins. In addition to the two PMUs configured for detecting inter-area modes, a total of 40 PMUs have been installed to the transmission network of England and Wales. The majority of them have been configured to report back to the central PDC at the ENCC, using the internal NG Business LAN.

In developing such a WAMS, reliable and high speed Information and Communication Technology (ICT) infrastructure that enables secure sharing of data among PMUs, PDCs and smart grid applications play an important role. The excess delays in the communication network are a challenging factor that affects the PMUs data transmission and could make the applications at best inefficient and at worse ineffective; especially for the control and protection applications where the delay should be sufficiently low in order to act in time. A Wide Area Network (WAN) is more than just end user devices. It comprises of links, routers, protocols, mechanisms, etc. that when all these components are considered, setting up a WAN can be a complex and costly deal. The extent of WAN makes the direct experiment almost impossible. Apart from the economic issues, it can lead to a serious damage and loss of data. Hence there is a need to have simulation models and testbeds which can accurately imitate the network behavior. By simulating the intended network, it is possible to test the newly proposed mechanisms, protocols, topologies, etc. or modify some network parameters and observe the effect before actual deployment [6].
This paper uses an existing WAMS scenario installed on the GB transmission system to evaluate the performance of WAN communications. The delay of the considered WAN will be fully investigated for various protocols, mechanisms, and future developments using both proprietary and open source DES, OPNET [7] and OMNeT++ [8]. The rest of the paper is structured as follows: Section II provides information about the physical structure and characteristics of the considered WAN model. Section III presents the simulation of the WAN communications network for different scenarios using OPNET. Section IV presents the same network simulation using $\mathrm{OMNeT++}$. Finally, the paper is concluded in Section V.

\section{Wide AreA Network Model ARChitecture}

The real WAN model used in this paper consists of 9 substations, which are geographically distributed. Except for substations 8 and 9, which have been equipped with two PMUs, all other substations have only one PMU. These PMUs obtain the analog input signals corresponding to voltages and currents from the instrument transformers and measure power system parameters. PMUs are connected to a Local Area Network (LAN) and the LAN is in turn connected via a substation router to the WAN. Using this network the measurement data from the PMUs are transmitted to a PDC server located at the data centre. Fig. 2 presents a simplified schematic of the WAN model infrastructure. Substations 6 and 7 are connected to the WAN through $2 \mathrm{Mbps}$ links and the other substations are connected by $256 \mathrm{Kbps}$ links. In addition, bandwidth of the link between the IP cloud and PDC is equal to $155 \mathrm{Mbps}$. Links are shared and used for different communication applications.

All PMUs have a sampling rate of 50 samples per second so they generate constant traffic. However, there are other substation based applications that generate variable traffic. The PMU in substation 1 is Arbiter 1133A and the size of packets

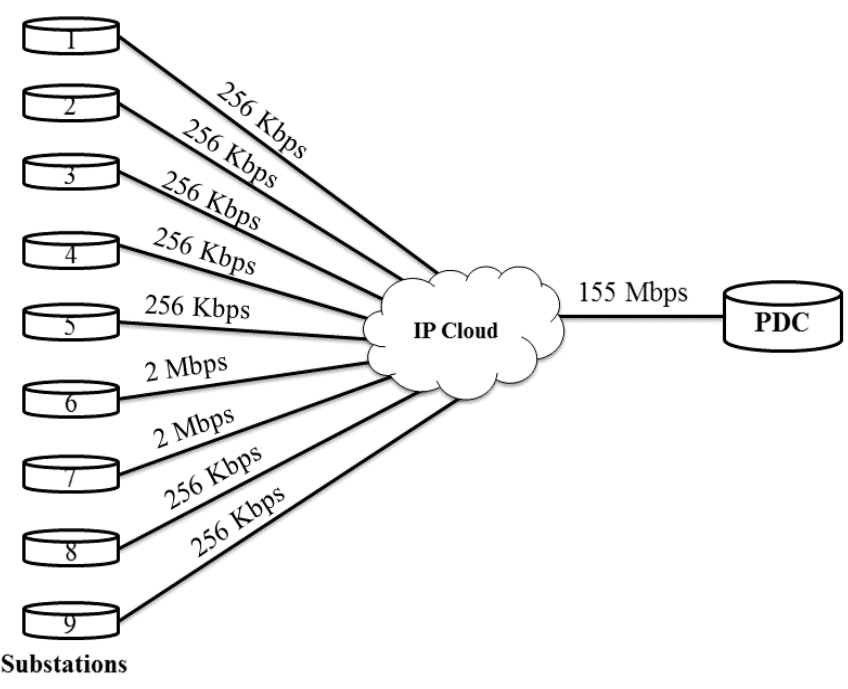

Fig. 2. WAN infrastructure schematic of the real WAMS 
transmitted from it is equal to 50 bytes [8]. While for the other 8 substations AMETEK TR-2000 multi-function recorder is used as a PMU and generates packets with the size of 42 bytes [10]. The packet sizes were obtained by running Wireshark [11] on the Psymetrix PDC server and capturing PMUs packets. This allows intercepting and displaying details of synchrophasor and other application generated packets being transmitted or received over the network interface. Therefore, we can have the packet specification of all the PMUs that are communicating with the PDC. The packet size depends on the number of synchrophasor parameters that a PMU measures. It should be also noted that the PDC uses DELL PowerEdge with $1 \mathrm{Gbps}$ connection ports as a server and as mentioned the deployed PMUs are not all from the same manufacturer. PMUs generated packets are transmitted to the PDC server based on TCP/IP protocol in the actual installed WAMS. However, in our analysis other communication protocols, mechanisms, and topologies will be investigated for future developments.

According to the information provided by NG, substations 6 and 7 which are connected to the WAN by 2 Mbps links, have more communications network activity in terms of staff presence and data transferred. Whereas substations 8 and 9, which have been equipped with two PMUs, have a lower level of background traffic. Based on this information, some substations need to be modelled differently from others. Due to a lack of detail regarding some aspects of the model and also for simplicity, some assumptions and simplifications have been made in order to perform the simulations. All substations have one local server and several workstations. Substations 1 to 5 are assumed to have a similar structure and each of them has five workstations such that one of them is defined to operate as a PMU. Substations 6 and 7 are assumed to have 13 workstations each such that one of the workstations works as a PMU. Finally, substations 8 and 9 have 3 workstations each such that two of them work as a PMU. Furthermore, background traffic should be specified for the links between substations and data centre in the WAN. The determined background traffic of each link is proportional to the number of workstations in different substations and the traffic they generate. Table I shows the

TABLE I

WAN LINKS BACKGROUND TRAFFIC

\begin{tabular}{ccc}
\hline \hline From & To & $\begin{array}{c}\text { Background traffic } \\
\text { (Percentage of link bandwidth) }\end{array}$ \\
\hline Substation 1 & IP cloud & $50 \%$ \\
Substation 2 & IP cloud & $50 \%$ \\
Substation 3 & IP cloud & $50 \%$ \\
Substation 4 & IP cloud & $50 \%$ \\
Substation 5 & IP cloud & $50 \%$ \\
Substation 6 & IP cloud & $70 \%$ \\
Substation 7 & IP cloud & $70 \%$ \\
Substation 8 & IP cloud & $0 \%$ \\
Substation 9 & IP cloud & $0 \%$ \\
IP cloud & Data Center & $60 \%$ \\
\hline \hline
\end{tabular}

considered background traffic level for WAN links in terms of percentage of link bandwidth. It is not possible to simulate the exact data traffic of the real network due to its stochastic nature. However, reasonably accurate traffic profiles should be determined and adopted for implementation.

The overall communication delay consists of four components: Transmission delay, which occurs during transmission and depends on the data size and link data rate; Propagation delay, which is related to the transmission distance and speed of the employed media; Processing delay, which is the time taken to process the packets, for instance in routers; Finally, queuing delay that is caused by the network congestion and is the time packets need to wait in a queue until they can be processed [12]. However, it is also necessary to include the internal processing delay of the PMUs in the modelled network in order to have the delay from the PMUs time-stamp point until packets reach the PDC server. Investigations were carried out to accurately estimate the internal delay of the two types of PMUs according to their configuration settings, operation manuals, and provided information from the manufacturers. The Arbiter PMU installed in substation 1 configured in a way to provide more accurate measurements, while AMETEK PMUs installed in other substations configured to provide measurments quickly. Based on the investigations, in the case of Arbiter PMU the average delay of $95 \mathrm{~ms}$ and for AMETEK PMUs the average delay of $30 \mathrm{~ms}$ have been calculated that are taken into consideration in the simulation model.

\section{NETWORK SIMULATION USING OPNET MODELER}

The DES tool that we have used in this section is the OPNET Modeler, Optimized Network Engineering Tools [7]. It provides advanced communications network modelling and simulation capabilities. OPNET provides a huge library of models and commercially available network technologies. Its friendly Graphical User Interface (GUI) and flexibility make the model building and implementation phases easier. It has a hierarchical modelling procedure that is divided into three main scopes, which are network, node, and process models [14]. Network devices such as workstations, switches and routers are called nodes. A node model consists of modules connected by packet streams or statistical wires and each module is assigned to a process model to achieve the required behaviour [15].

Fig. 3 illustrates the simulation model as implemented using OPNET [7]. The 9 octagons on the left-hand side of IP cloud represent the 9 substations and the one on right-hand side is the data centre. The geographical locations of substations and data centre are not their actual locations in the real system. The workstation nodes are Ethernet_wkstn_adv model as defined in the OPNET model library [7]. It is important to note that there are different methods available in OPNET for introducing communications network traffic. Since the EtE delays of PMU packets from the source application layer to destination 


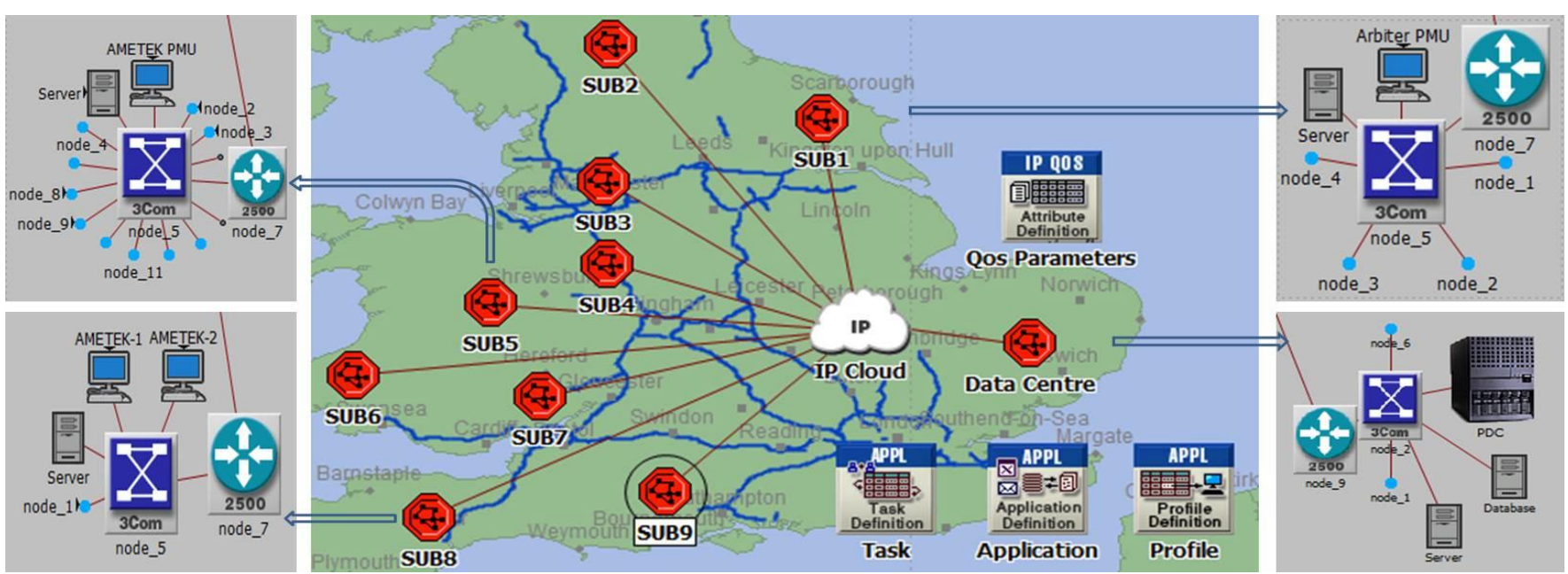

Fig. 3. Simulated network in OPNET Modeler

application layer are required in order to accurately model PMU, traffic custom applications were defined. In OPNET, all custom applications are defined through a series of tasks. Each task is further divided into individual phases [16]. Therefore, two tasks were configured by using the Task Config utility object in OPNET that represent traffic associated with the two types of PMUs that exist in the model. The main difference between these two tasks is the packet size such that in the case of Arbiter PMU is 50 bytes and for AMETEK PMU is 42 bytes. Both tasks were configured to generate packets every $20 \mathrm{~ms}$, which means they create 50 samples per second. The final destination of all PMUs packets is a DELL PowerEdge server in the data centre node. After defining the single phase task of custom application for both types of PMUs, the custom application itself can be configured using the Application Config utility object in OPNET. Finally, by using Profile Config utility the profile that employs the configured custom applications can be specified. A user profile is a mechanism for specifying how applications are used by an end user during a simulation. In order to separate the latency results for each PMU a distinct profile was created for each individual PMU in the network. Furthermore, the calculated internal delays for the two PMU types were introduced in both their node models through the packet stream connected to the application layer.

Apart from PMUs, for other workstations inside the substations standard application models were used to configure traffic. Standard application models in OPNET provide an adequate level of detail for modelling commonly used applications. For these workstations, one profile consisting of Database Access, File Transfer, and Email applications were specified that may send data to the local server or the two servers at data centre. Once all applications and user profiles have been defined, they can be deployed by corresponding nodes. This can be done by configuring each node through the
Edit Attributes window to support desired profiles and applications.

The traffic is generated in the application module and then is passed to the transport layer module and thereafter to the network layer and so on. The PMU generated data will be first transmitted to the substation switch, and then to the substation router. Switches and routers were given the default configurations and the 100Base-T link was used for all substation LAN communication. The IP/Ethernet cloud was specified with normal distribution packet latency with the mean outcome of 0.03 seconds. Substations were connected to $I P /$ Ethernet cloud using PPP point-to-point links and the same type of link used for connecting IP/Ethernet cloud to the router in the data centre. For the links between substations and data centre in the WAN, data rates alongside their background traffic were defined according to the specified architecture in Section II. Finally, inside the data centre, through the router data will then be transmitted via LAN to the PDC server.

After the completion of network configuration, the statistics to be collected can be specified in OPNET [7]. In this WAMS network research, the end to end delay from the PMU to PDC is a key statistic that reflects the WAMS performance. Therefore from the node statistics section in OPNET, the responding or requesting custom application statistics can be selected. It should be noticed that in OPNET the default collection mode for network delay statistics is bucket mode. In this mode OPNET groups data points that occur within a period, referred to as a bucket, and then applies a statistical function to each group of values. The resulting output vector contains one value for each bucket. This value can be the maximum, minimum, mean, etc. of the results for samples available in a bucket. The collection mode was also changed to All Values mode to obtain all the generated PMUs samples latency in order to calculate various characteristics of OPNET results accurately. 


\section{A. TCP/IP Protocol}

TCP, Transmission Control Protocol, is a transport mechanism over IP, which offers a connection oriented communication. It supports retransmit capabilities, flow control, buffer handling and traffic shaping properties. TCP/IP is used by common services like FTP, Database, HTTP, etc. TCP rearranges data packet in the specified order and retransmits lost or corrupted data. In order to handle packet drops, every packet being received is acknowledged back to inform sender the successful transmission of packet. Afterwards, sender will release the transmit buffer for new data to be transmitted. TCP provides reliable communication by retransmitting of the lost or corrupted data. However, the acknowledgment and retransmission features lead to excessive delays [17].

Table II presents the latency characteristics of OPNET results including maximum, minimum, average, and standard deviation when PMUs packets are transmitted based on TCP/IP protocol. Furthermore, other scenarios have been analyzed to evaluate the WAN performance for the future WAMS developments. In one of the scenarios, the latencies of the PMUs packets have been compared when different number of PMUs are deployed in substations and for different level of WAN links background traffic. As the WAN shares links for different applications, the ICT infrastructure of the WAMS has been tested for various background traffic conditions. Table III shows the obtained simulation results. It can be seen that the performance of the system in higher links background traffic and number of PMUs is degraded significantly. In this case phasor data have to be queued in the router buffer and wait for processing due to the network congestion. In another scenario, the number of substations has been increased in order to investigate the performance of WAN, when more substations are equipped with PMUs and join the WAMS. It has been assumed that the new connected substations have the same structure as substations 1 to 5 in the considered WAMS. Hence they have been equipped with one PMU and connected to the WAN through $256 \mathrm{kbps}$ links. Table IV shows the average

TABLE II

TCP/IP SCENARIO

LATENCY CHARACTERISTICS OF OPNET RESULTS (ms)

\begin{tabular}{cccccc}
\hline \hline \multirow{2}{*}{ SUB } & PMU & Min & Max & Average & STDEV \\
\hline 1 & 1 & 127.8 & 403.3 & 165.1 & 28.73 \\
\hline 4 & 1 & 64.85 & 354.9 & 100.67 & 29.06 \\
\hline 5 & 1 & 65.12 & 525.7 & 104.94 & 50.38 \\
\hline 7 & 1 & 62.15 & 120.9 & 86.04 & 8.39 \\
\hline \multirow{2}{*}{8} & 1 & 60.49 & 204.2 & 82.82 & 9.39 \\
& 2 & 56.03 & 201.2 & 81.73 & 9.42 \\
\hline \multirow{2}{*}{9} & 1 & 61.53 & 218.4 & 84.49 & 9.5 \\
& 2 & 60.67 & 202.2 & 83.76 & 9.35 \\
\hline \hline
\end{tabular}

TABLE III

DIFFERENT NUMBER OF PMUS AND LINKS BACKGROUND TRAFFIC AVERAGE LATENCY (ms)

\begin{tabular}{ccccc}
\hline \hline \multirow{2}{*}{ Backgroud Traffic } & Number of PMUs & SUB 1 & SUB 5 & SUB 7 \\
& 1 & 148.6 & 82.3 & 80 \\
\multirow{2}{*}{$0 \%$} & 2 & 150.6 & 83.8 & 80.7 \\
& 3 & 152.9 & 84.8 & 82.1 \\
& 4 & 153.9 & 86.3 & 82.3 \\
\hline \multirow{2}{*}{$20 \%$} & 1 & 150.6 & 87.3 & 80.3 \\
& 2 & 153.7 & 88.8 & 81.3 \\
& 3 & 158.3 & 91.3 & 82.3 \\
& 4 & 174 & 101.1 & 82.7 \\
\hline \multirow{2}{*}{$40 \%$} & 1 & 157.5 & 92.4 & 81.6 \\
& 2 & 165.1 & 97.4 & 82.2 \\
& 3 & 207.4 & 133.6 & 82.7 \\
& 4 & 2993.9 & 3512.9 & 84 \\
\hline \multirow{2}{*}{$60 \%$} & 1 & 176.5 & 107.4 & 83.7 \\
& 2 & 363.2 & 176.3 & 84.1 \\
& 3 & 3776.5 & 3603.8 & 84.9 \\
& 4 & - & - & 85 \\
\hline \hline
\end{tabular}

TABLE IV

INCREASE THE NUMBER OF SUBSTATIONS AVERAGE LATENCY (ms)

\begin{tabular}{cccc}
\hline \hline \multicolumn{3}{c}{$(\mathrm{ms})$} & \\
\hline $\begin{array}{c}\text { Number of Additional } \\
\text { Substations }\end{array}$ & SUB 1 & SUB 5 & SUB 7 \\
\hline 10 & 172.7 & 106.2 & 90.3 \\
\hline 15 & 178.5 & 108.6 & 93.4 \\
\hline 20 & 188.8 & 118.8 & 105 \\
\hline
\end{tabular}

latency of the PMUs for three of the substations when extra substations are added to the WAMS. After connecting more than 20 extra substations the latency will be very high and from the point we add $25^{\text {th }}$ substation (total of 34 substations) the communication network will become unstable and latency values are not converged.

\section{B. UDP/IP Protocol}

UDP, User Datagram Protocol, is a transport mechanism over IP, which offers a connection-less communication. It does not provide mechanism for flow control and rate adaptation that otherwise is associated with TCP. UDP can be used for unicast, multicast, broadcast and anycast applications. It is used by services like VOIP, DNS, DHCP, etc [17]. In the case of UDP there is no built-in ordering and recovery of data, but the transmission speed is higher than TCP and it can provide stable delay. Therefore, time-sensitive applications often use UDP since a small amount of lost data is preferable over delayed data [18]. In this scenario, all the PMUs have been configured to use UDP/IP protocol in their communications with PDC. Table $\mathrm{V}$ presents the simulation results. The obtained results show a lower average latency compared to TCP/IP protocol. For further improvement of the WAN performance, in the next 
TABLE V

UDP/IP SCENARIO

LATENCY CHARACTERISTICS OF OPNET RESULTS (ms)

\begin{tabular}{cccccc}
\hline \hline \multirow{2}{*}{ SUB } & PMU & Min & Max & Average & STDEV \\
\hline 1 & 1 & 124.6 & 355 & 160.3 & 26.34 \\
\hline 4 & 1 & 59.78 & 369.8 & 95.38 & 27.48 \\
\hline 5 & 1 & 59.9 & 285.4 & 95.82 & 25.18 \\
\hline 7 & 1 & 57.55 & 124.7 & 82.74 & 8.86 \\
\hline \multirow{2}{*}{8} & 1 & 57.9 & 218.9 & 80.76 & 9.43 \\
& 2 & 60.23 & 210.1 & 80.78 & 9.46 \\
\hline \multirow{2}{*}{9} & 1 & 57.72 & 189.8 & 80.19 & 9.33 \\
& 2 & 59.97 & 196.9 & 81.38 & 9.22 \\
\hline \hline
\end{tabular}

scenario we investigate the deployment of Quality of Service (QoS) mechanism.

\section{UDP/IP Protocol with QoS}

QoS policy can be used to ensure the excessive delay does not occur for the time-critical applications packets in a shared network. In fact, QoS is a request from an application to the network to provide a guarantee on the quality of a connection. By marking packet for different levels of priority, the queue with higher priority is first checked for sending packet. Fig. 4 illustrates how this classification is implemented in a router [17]. The Weighted Fair Queuing (WFQ) scheduling schemes alongside the Type of Service (ToS) profile have been used in OPNET to carry out the relevant analysis. The QoS profiles in OPNET contain detailed information about their mechanism settings such as the number of queues used, their respective weights, and queue size limits.

Table VI shows the simulation results. According to the obtained results QoS mechanism improved the WAN performance significantly, especially regarding maximum latency and deviation. However, a slight increase in the average latency for PMU 2 in substation 9 can be seen. This happens because of the effect that the traffic in the same priority class has on themselves.

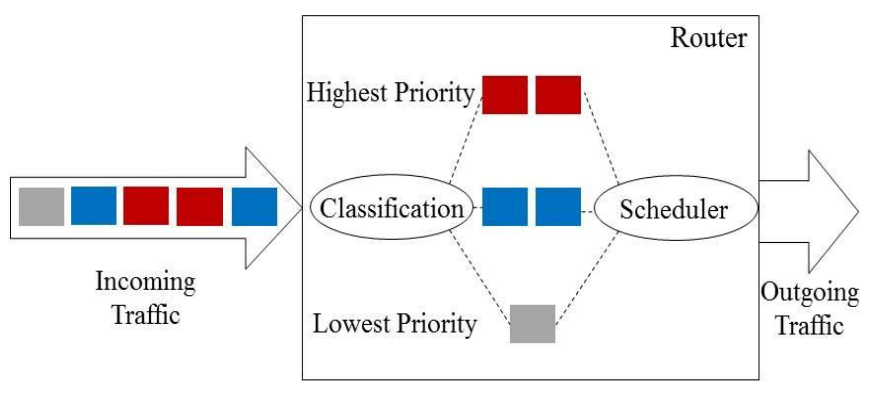

Fig. 4. Classification of traffic based on priority
TABLE VI

UDP/IP WITH QOS SCENARIO

LATENCY CHARACTERISTICS OF OPNET RESULTS (ms)

\begin{tabular}{cccccc}
\hline \hline \multirow{2}{*}{ SUB } & PMU & Min & Max & Average & STDEV \\
\hline 1 & 1 & 125.9 & 212.6 & 147.57 & 9.44 \\
\hline 4 & 1 & 62.02 & 153.6 & 82.23 & 9.68 \\
\hline 5 & 1 & 61.5 & 142.8 & 82.44 & 9.46 \\
\hline 7 & 1 & 59.08 & 117.7 & 79.37 & 8.11 \\
\hline \multirow{2}{*}{8} & 1 & 60.8 & 142.3 & 79.6 & 8.3 \\
& 2 & 57.84 & 143.6 & 80.07 & 8.65 \\
\hline \multirow{2}{*}{9} & 1 & 56.57 & 141.3 & 80.2 & 8.72 \\
& 2 & 62.9 & 144.3 & 84.5 & 8.5 \\
\hline \hline
\end{tabular}

\section{NeTwORK Simulation USING OMNET++}

The open source DES tool OMNeT++, Objective Modular Network Testbed in $\mathrm{C}++$, has also been used to simulate the considered WAN [8]. OMNeT++ is modular component-based simulation package with GUI support. It has generic and flexible architecture that enables to easily integrate various modules [20]. Along with the OMNeT++ widespread application more models, networks, protocols etc. are contained in model library. The INET framework, an open-source communication network simulation package for the OMNeT++, contains the common node models and protocols [19]. However, minor modifications have been made to simulate the WAN that will be discussed in this section.

Model components are described and assembled in a high-level domain-specific language called NED in OMNeT++. Using NED Editor the communication network's topology is created. The NED can be edited both graphically and in text mode. The network description consists of a number of component descriptions such as channels, simple and compound module types. To simulate the considered WAN, the StandardHost node model in INET framework has been used for workstations. In order to model the two types of PMUs, the internal delays of them have been added by modifying the StandardHost node model. Furthermore, the required communication links according to their data rates have been defined. Using these workstations and communication links, different compound modules to represent different substations and data centre have been designed. By connecting these compound modules the whole network can be created as shown in Fig. 5. For IP cloud the InternetCloud node model in INET framework has been used. It has been configured based on the specified parameters in Section III for OPNET and by writing a separate XML file.

In OMNeT++, introducing communications network traffic for each workstation takes place in ini files that also carry configuration options for the simulator. In this section, it is assumed that PMUs are communicating with PDC over 


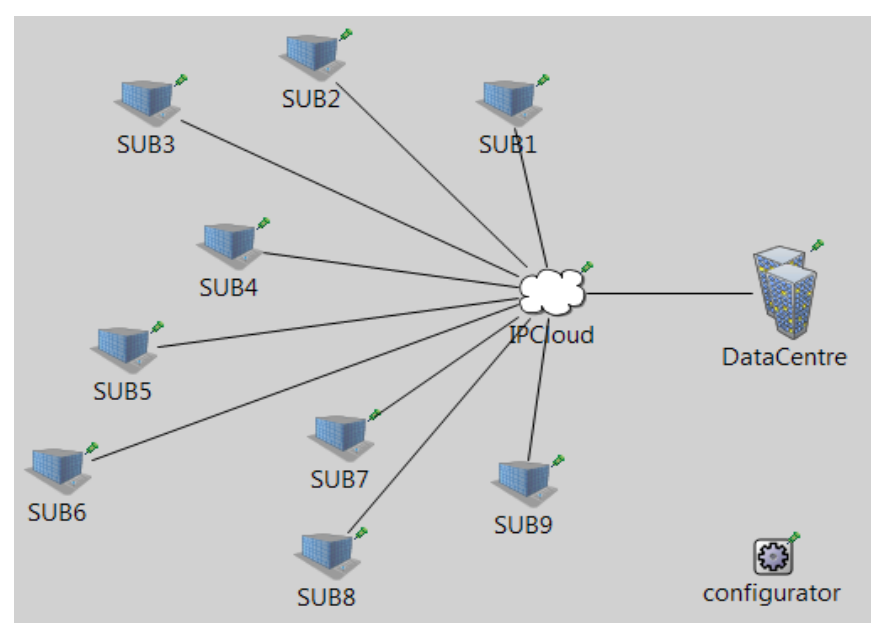

Fig. 5. Simulated network in OMNeT++

UDP/IP protocol. From the application sources available in INET framework, the UDPBasicApp has been used to configure the two types of PMUs traffic. In order to have the EtE delay of each PMU separately in the simulation result, the PMUs have been configured to send their data to the different ports of the PDC node. On the other hand, the UDPSink application has been defined for PDC to handle and discard the transmitted PMUs packets, as well as calculating the EtE delays. Apart from the PMUs, for other workstations inside the substations and data centre, traffic similar to OPNET simulation has been specified using TCPBasicClientApp. After assigning traffic and configuration options for simulation in the ini file, the simulation can be run. Simulation results are saved in the results folder of the project. The OMNeT++ Analysis Tool enables user to select, process and plot the results. It will save the analysis into a .anf file in the parent folder. Table VII shows the latency characteristics from the OMNeT++ obtained results. It shows a similar behavior with OPNET results, especially in the case of average values. However, there are some differences that should be investigated more. The

TABLE VII

UDP/IP SCENARIO

LATENCY CHARACTERISTICS OF OMNET++ RESULTS (ms)

\begin{tabular}{cccccc}
\hline \hline \multirow{2}{*}{ SUB } & PMU & Min & Max & Average & STDEV \\
& 1 & 128.04 & 303.09 & 156.3 & 30.5 \\
\hline 1 & 1 & 62.72 & 234.27 & 99.4 & 34.5 \\
\hline 4 & 1 & 62.46 & 240.8 & 96.3 & 36.9 \\
\hline 5 & 1 & 60.65 & 258.6 & 83.83 & 24.2 \\
\hline 7 & 1 & 64.20 & 136.65 & 76.52 & 18.1 \\
\multirow{2}{*}{8} & 2 & 68.8 & 140.8 & 81.25 & 18.1 \\
\hline \multirow{2}{*}{9} & 1 & 64.14 & 136.3 & 77.44 & 18.59 \\
& 2 & 68.9 & 141.3 & 82.15 & 18.57 \\
\hline \hline
\end{tabular}

differences can be due to the model components library and the way that communication links background traffic has been defined in OMNeT++, by reducing data rates of the communication links.

\section{CONCLUSION}

WAMS applications inherently depend on the underlying ICT infrastructures. Even if all the phasors are successfully delivered to PDC, the time delay associated with each phasor measurement makes the derived system state to be different from the actual system state by that time. In addition, for WAMS applications, especially control and protection, delay needs to be sufficiently low in order to act in a timely manner. Therefore, it is necessary to fully investigate the EtE delay occurs in WAMS. There are numerous DES tools available for the analysis of the performance and behavior of communication networks. In this paper both proprietary and open source DES tools have been used to simulate an existing WAMS that has been installed on the transmission system of GB. Various scenarios were considered for analyzing possible future developments as well as methods to improve the performance. Further work will be focused on refinement of the WAMS communication model. Also consideration will be given to other delay sources in the system, such as PDC processing delays.

\section{ACKNOWLEDGMENT}

The authors would like to thank National Grid for the provision of information on their communications infrastructure and also Psymetrix for their assistance in obtaining the TCP traffic information from the actual WAMS.

\section{REFERENCES}

[1] P. M. Ashton, G. A. Taylor, M. R. Irving, A. M. Carter, M. E. Bradley, "Prospective Wide Area Monitoring of the Great Britain Transmission System using Phasor Measurement Units," Power and Energy Society 2012 IEEE General Meeting, 22-26 July 2012.

[2] K. Gajrani, A. Bhargava, K. G. Sharma, R. Bansal, "Dynamic performance improvement of wind power integrated grid using TCP/IP communication network," 2013 IEEE Conference on Clean Energy and Technology (CEAT), pp. 405-410, 18-20 Nov. 2013.

[3] M. Golshani, G. A. Taylor, I. Pisica, P. Ashton, "Investigation of open standards to enable interoperable wide area monitoring for transmission systems, " 47th International Universities Power Engineering Conference (UPEC), 4-7 Sept. 2012.

[4] "Real-Time Application of Synchrophasors for Improving Reliability," NERC, 18 Oct., 2010, online: http://www.nerc.com.

[5] PhasorPoint, Psymetrix, UK, 2014.

[6] M. R. Christhu, N. Marium, J. Major, D. Shibin, "A comprehensive overview on different network simulators," International Journal of Engineering and Technology (IJET), vol. 5, no. 1, pp. 325-332, Feb-Mar 2013.

[7] OPNET Modeler, OPNET Technologies, [www.opnet.com]

[8] OMNeT++ Network Simulation Framework, [www.omnetpp.org]

[9] Arbiter Systems, [http://www.arbiter.com]

[10] AMETEK Power Instruments, [http://www.ametekpower.com]

[11] Wireshark, [http://www.wireshark.org] 
[12] P. Kansal, A. Bose, "Bandwidth and Latency Requirements for Smart Transmission Grid Applications," IEEE Transactions on Smart Grid, vol. 3, no. 3, pp. 1344-1352, Sept. 2012.

[13] X. Cheng, Y. Wang, Y. Ni, "The Study on the Communication Network of Wide Area Measurement System in Electricity Grid," 2012 International Conference on Solid State Devices and Materials Science, vol. 25, pp. 1708-1714, 2012.

[14] OPNET Modeler Documentation Set, Version 17.1.

[15] T.S. Sidhu, Y. Yin, "Modelling and Simulation for Performance Evaluation of IEC61850-Based Substation Communication Systems," IEEE Transactions on Power Delivery, vol. 22, no. 3, pp. 1482-1489, July 2007.

[16] A. S. Sethi, V. Y. Hnatyshin, "The Practical OPNET User Guide for Computer Network Simulation,” Boca Raton, USA: CRC Press, 2013.

[17] C. F. M. Danielson, L. Vanfretti, M. S. Almas, Y. Choompoobutrgool, J. O. Gjerde, "Analysis of communication network challenges for synchrophasor-based wide-area applications," Bulk Power System Dynamics and Control - IX Optimization, Security and Control of the Emerging Power Grid (IREP), 2013 IREP Symposium , pp. 1-13, 25-30 Aug. 2013.

[18] "A Guide for PMU Installation, Commissioning and Maintenance — Part II PMU Installation Procedures," NASPI, 5 June 2007, [Online]. Available: https://www.naspi.org

[19] INET Framework, [http://inet.omnetpp.org]

[20] T. Peter, G. Marek, F. Tomas, G. Radoslav, L. Michal, "Simulation of IMS using current simulators," 50th International Symposium ELMAR, vol. 2, pp. 545-548, 10-12 Sept. 2008. 